\title{
Effects of Amino Acid-Supplemented Diets on Sister Chromatid Exchange Frequency of Rats Treated with Cyclophosphamide
}

\author{
Jia F. Chen and Yue J. Lin \\ Department of Biological Sciences, St. John's University, \\ Jamaica, New York 11439, U.S.A.
}

Accepted September 24, 1986

The effects of unbalanced diets on sister chromatid exchange (SCE) frequency of rats were first reported by Chen and Lin (1986) who found that the SCE frequency of bone marrow was significantly higher in those rats fed on unbalanced diets, Diets $\mathrm{G}$ and Z. Diet $\mathrm{G}$ consisted of $75 \%$ Purina Rodent Chow 5001 (PRC) and $25 \%$ gelatin which lacked tryptophan. Diet $\mathrm{Z}$ consisted of $75 \% \mathrm{PRC}$ and $25 \%$ zein which contained no lysine and tryptophan.

If a diet is low in a specific amino acid, other amino acids present in the diet are not as completely utilized. This specific amino acid is considered to be a limiting amino acid in the diet (Morrison et al. 1961). The fasting plasma profile equivalent (FPPE) concept (Jarowski and Pytelwski 1975, Dubroff et al. 1979) was used to determine the order of limiting amino acids. FPPE value is determined by the following formula: (m moles of amino acid per $100 \mathrm{~g}$ of food) divided by ( $\mathrm{m}$ moles of amino acid per liter of animal's fasting plasma). The FPPE values of 10 essential amino acids in the Diet $P$ (i.e. PRC, control) and Diets $G$ and $Z$ have been measured and there is a good correlation between SCE frequency and the degree of variation among the FPPE values of amino acids in the diets (Chen and Lin 1986).

In this study, we added amino acids to the regular Diet $P$ and to a less balanced diet, Diet $G$, so that the differences among the FPPE values of essential amino acids are narrowed. It was found that the addition of limiting amino acids could reduce SCE frequency of bone marrow in rats treated with a mutagen, cyclophosphamide, especially at a higher dosage of cyclophosphamide (CP). The SCE frequency of bone marrow in rats on amino acid-supplemented Diet GAA was found to be significantly lower than those on Diet G.

\section{Materials and methods}

\section{Amino acid supplementation}

The calculation of FPPE values of essential amino acids in Diet $P$ and Diet $G$ was shown in a previous paper (Chen and Lin 1986). For convenience, the FPPE values of amino acids in Diets $P$ and $G$ are also included in Table 1 of this paper. To supplement a diet with more amino acids, an amino acid with a certain FPPE value is selected, and those amino acids with lower FPPE values are supplemented so that their FPPE values are the same as that of the selected amino acid. In Diet P, lysine has the lowest FPPE value (24.96) and is therefore the first limiting amino acid. The next limiting amino acid is tryptophan (26.30). If we are to add the limiting amino acids up to the histidine level (57.10), the amount of lysine ( $\mathrm{g}$ ) needed for $100 \mathrm{~g}$ of Diet $\mathrm{P}$ is: (histidine $\left.\mathrm{FPPE}_{\mathrm{FP}}-\mathrm{lysine}_{\mathrm{FPPE}}\right) \times($ plasma concentration of lysine) $\times$ (molecular weight of lysine), i.e. $(57.10-24.96) \times\left(0.389 \times 10^{3}\right) \times(146.2)=1.83 \mathrm{~g} . \quad$ In this study, the special Diet 3AA is Diet $\mathbf{P}$ supplemented with three limiting amino acids, lysine, tryptophan and threonine, so that the FPPE values of these three amino acids are the same as that of histidine, 57.10, and Diet GAA is Diet $G$ supplemented with the same three limiting amino acids. The FPPE values of amino acids in Diets $3 A A$ and GAA are in Table 1. 


\section{Cyclophosphamide treatments}

Young male Sprague-Dawley rats $(2 \mathrm{n}=42,6$ weeks old, about $225 \mathrm{~g}$ in weight) were housed two in a cage and allowed a one-week acclimation period. There were two groups of rats, 3AA and GAA (12 rats each), on the amino acid-supplemented diets for four weeks. They were allowed food and water ad libitum. The overall condition of the rats during the course of the study was monitored by weekly measurement of the food intake and body weight. The average food intake and body weight increase were found to be similar among the 2 groups and those groups in the previous study.

\section{Slide preparation}

The preparation of slides was described elsewhere (Chen and Lin 1986).

Table 1. Fasting plasma profile equivalent values of essential amino acids in various diets based on the fasting plasma profile of Sprague-Dawley rats

\begin{tabular}{lllllllllll}
\hline $\begin{array}{c}\text { Essential } \\
\text { amino acid }\end{array}$ & Trp & Thr & ILeu & Leu & Lys & $\begin{array}{c}\text { Met }+ \\
\frac{1}{2} \text { Cys }\end{array}$ & $\begin{array}{l}\text { Phe }+ \\
\text { Tyr }\end{array}$ & Val & Arg & Hist \\
\hline FPPE value & & & & & & & & & \\
Diet P & 26.30 & 38.39 & 149.80 & 129.60 & 24.96 & 106.66 & 83.48 & 102.30 & 54.25 & 57.10 \\
Diet G & 19.81 & 48.94 & 155.67 & 153.10 & 37.33 & 108.46 & 94.17 & 127.82 & 118.01 & 62.90 \\
Diet 3AA & $57.10^{\mathrm{a}}$ & $57.10^{\mathrm{a}}$ & 149.80 & 129.60 & $57.10^{\mathrm{a}}$ & 106.66 & 83.48 & 102.30 & 54.25 & $57.10^{\mathrm{a}}$ \\
Diet GAA & $62.90^{\mathrm{a}}$ & $62.90^{\mathrm{a}}$ & 155.67 & 153.10 & $62.90^{\mathrm{a}}$ & 128.46 & 94.17 & 127.82 & 118.01 & $62.90^{\mathrm{a}}$ \\
\hline
\end{tabular}

a Amino acid added to histidine level (FPPE)

Table 2. Effects of cyclophosphamide and diets on SCE in rats

\begin{tabular}{cccc}
\hline \hline \multirow{2}{*}{ Diet } & \multicolumn{3}{c}{ Cyclophosphamide treatment } \\
\cline { 2 - 4 } & $0 \mathrm{mg}$ & $1 \mathrm{mg}$ & $5 \mathrm{mg}$ \\
\hline P** $^{* *}$ & $4.78 \pm 2.71 @$ & $7.15 \pm 2.63^{*}$ & $24.81+6.15^{*}$ \\
G $^{* *}$ & $10.27 \pm 4.76^{*}$ & $14.88 \pm 4.73^{* *}$ & $35.37 \pm 6.97^{* *}$ \\
GA & $8.81+3.57^{*}$ & $9.25 \pm 4.23^{*}$ & $19.17 \pm 5.80^{* *}$ \\
\hline
\end{tabular}

(a) Mean \pm Standard deviation

* Denotes significant difference from the control (Diet P) at the same level of CP treatment (Student ttest at $5 \%$ level)

* Denotes significant difference from the control $(0 \mathrm{mg})$ within a diet group (Student $\mathrm{t}$-test at $5 \%$ level)

** Results from Chen and Lin (1986)

Results

In a previous study (Chen and Lin 1986), an average of 4.78 and 10.27 SCE per cell were recorded in rats, receiving no $\mathbf{C P}$, on Diet $P$ and Diet $G$ respectively (Table 2). In this study, rats on the two diets supplemented with three limiting amino acids, Diet 3AA and Diet GAA, without CP treatment had similar SCE frequencies (Table 2, Figs. 1, 2).

$\mathrm{CP}$ treatment increased SCE frequency in all rat groups. The higher the CP dosage, the higher the SCE frequency (Table 2). After CP treatment at $1 \mathrm{mg} / \mathrm{kg}$ body weight (b.w.), the SCE frequencies of Groups 3AA (9.25, Fig. 3) and GAA (11.17, Fig. 4) were higher than that of Group P (7.15), but were significantly lower than that of Group G (14.88). When treated at $5 \mathrm{mg} / \mathrm{kg}$ b.w., the SCE frequencies of Groups 3AA (19.17, Fig. 5) and GAA (21.17, Fig. 6) were lower than those of Group P (24.81) and Group G (35.37). It appears that addition of amino 
acids, based on FPPE concept, reduced SCE frequency especially at higher CP dosage (5 mg/ kg b.w.).

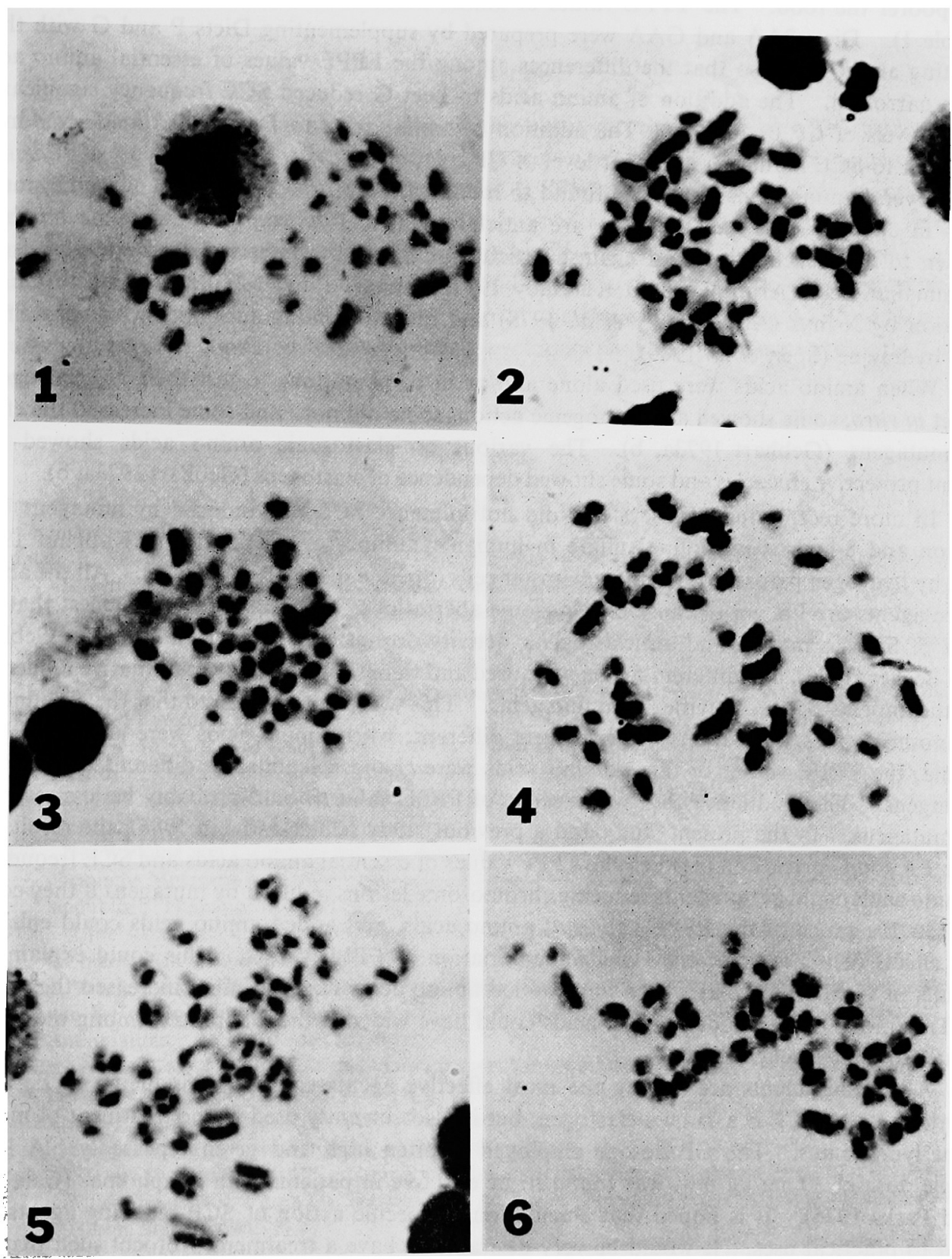

Figs. 1-6. Bone marrow chromosomes of rats on two special diets, Diet 3AA and Diet GAA, showing various frequencies of sister chromatid exchange. 1, Diet 3AA, no cyclophosphamide treatment, $\mathrm{SCE}=8 . \times 1550$. 2, Diet GAA, no cyclophosphamide $(\mathrm{CP})$ treatment, $\mathrm{SCE}=4$. $\times 1700$. 3, Diet $3 \mathrm{AA}, 1 \mathrm{mg}$ of CP per kg body weight (b.w.), SCE $=11 . \quad \times 1550 . \quad 4$, Diet GAA, $1 \mathrm{mg}$ of CP per kg b.w., $\mathrm{SCE}=11 . \times 1700 . \quad 5$, Diet $3 \mathrm{AA}, 5 \mathrm{mg}$ of $\mathrm{CP}$ per $\mathrm{kg}$ b.w., $\mathrm{SCE}=16$. $\times 1700$. 6, Diet GAA, $5 \mathrm{mg}$ of CP per kg b.w., $\mathrm{SCE}=19 . \quad \times 1300$. 


\section{Discussion}

According to the FPPE theory, the wider the variation in its FPPE values of amino acids, the poorer the food. The FPPE values of amino acids in Diet $G$ varied with a wide range (Table 1). Diets 3AA and GAA were prepared by supplementing Diets $P$ and $G$ with three limiting amino acids so that the differences among the FPPE values of essential amino acids were narrowed. The addition of amino acids to Diet $\mathrm{G}$ reduced SCE frequency significantly at all levels of $\mathrm{CP}$ treatments. The addition of amino acids to Diet $\mathbf{P}$ also made rats more resistant to SCE induction at higher level of CP treatment.

Several amino acids have been found to reduce chromosome aberrations induced by mutagens in cultured cells and therefore are anticlastogenic. For example, L-cysteine has been shown to have such protection against structural chromosome aberrations by mutagens for mammalian cells (Arutunian and Kuleshov 1972, Gebhart 1969, 1970) and against SCE induction by X-rays (Abramovsky et al. 1978) and radical-forming substances, hydroxylamine and hydrazine (Speit et al. 1980).

When amino acids were used alone and/or in combinations to test their anticlastogenic effect in vitro, some showed anticlastogenic action, some did not, and some increased the effect of mutagens (Gebhart 1973a, b). The various anticlastogenic amino acids showed different protective efficacies and some showed dependence of clastogens (Gebhart 1973a, b).

In more recent studies, L-cysteine did not influence SCE rate induced by mutagens Trenimon and 8-hydroxyquinoline sulfate in human lymphocytes (Gebhart and Kappauf 1980) and by hydrogen peroxide in Chinese hamster cells (Speit et al. 1982) in cultures. All the above three agents are known to cause chromosome aberrations. Thus these results suggest that the sites of SCE formation and anticlastogenic activity do not coincide. In a study by Gebhart and Becher (1976), two different media were used and were shown to have quantitative influences on the anticlastogenic activities of amino acids. This was probably due to that the amounts of amino acids present in the two media were different; when amino acids were added to these media, the FPPE values of these amino acids were changed leading to different reactions to mutagens. The medium with a wider range of FPPE values would probably be less resistant to mutagens. In the present study and a previous study (Chen and Lin 1986), the results indicate a good correlation between the FPPE values of essential amino acids and SCE frequency. Amino acids could act as agents reducing chromosome lesions induced by mutagens if they could narrow the range of the FPPE values of amino acids, and added amino acids could enhance the effects of mutagens if they widen the variation of FPPE values. This could explain the results of Gebhart (1973a) where some added amino acids (e.g. arginine) increased the effects of Trenimon. These added amino acids could have widened the differences among the FPPE values of amino acids.

Alkylating agents are among the most effective agents capable of inducing SCE. The alkylating agent $\mathrm{CP}$ is a known clastogen, but is also currently used in the treatment of malignant lymphomas. The CP dosage employed is often high and given repeatedly. A large single dose of $30 \mathrm{mg} / \mathrm{kg} \mathrm{b.w}$. was found to be effective in patients with lymphomas (Calabresi and Parks 1975). It is hoped that elucidation of specific action of SCE-reducing agents can lead to practical uses. It would be very desirable to have a treatment protocol such that the antitumor drugs are still effective in chemotherapy while their toxicity can be reduced to the minimum. On the other hand, a decrease in serum blood used urea nitrogen in rat was observed through dietary supplementation of three limiting amino acids (Torre et al. 1981). With the use of lysine-supplemented diet, Jarowski and Pytelwski (1975) were able to demonstrate lower cholesterol levels in rats. Persons taking lysine and tryptophan supplementation and showing lower plasma cholesterol and triglyceride levels were also reported (Raja and 
Jarowski 1975). In these experiments, the amount of amino acids supplemented was calculated based on the fasting plasma profile equivalent concept. These results along with our results suggest that we might benefit from manipulation of dietary components in many different ways.

\section{Summary}

Less balanced diets are known to induce higher sister chromatid exchange (SCE) frequency in rats. The SCE frequency of various diet rat groups correlated well with the degree of variation among the fasting plasma profile equivalent (FPPE) values of amino acids in their diets. To narrow the differences among the FPPE values of essential amino acids, three limiting amino acids, lysine, tryptophan, threonine, were added to the regular diet (Diet $\mathrm{P}$ ) and to a less balanced diet, Diet $G$. The amounts of amino acid supplemented were calculated based on the FPPE concept. It was found that the addition of the three amino acids could reduce SCE frequency of those rats treated with a mutagen, cyclophosphamide (CP), especially at a higher dosage of CP.

\section{References}

Abramovsky, L., Vorsanger, G. and Hirschhorn, K. 1978. Sister chromatid exchange induced by X-ray of human lymphocytes and the effect of L-cysteine. Mut. Res. 50: 93-100.

Arutunian, R. M. and Kuleshov, N. P. 1972, Modification by cysteine of tio-TEF induced chromosome aberrations in cultured human leucocytes. Genetika (Moscow) 8: 148-153.

Calabresi, P. and Parks, R. E. Jr. 1975. Alkylating agents, antimetabolites, hormones, and other antiproliferative agents. In: The Pharmacological Basis of Therapeutics, 5th ed. eds. L. S. Goodman. and A. Gilman. Macmillan, New York, pp. 1245-1307.

Chen, J. F. and Lin, Y. J. 1986. Effects of cyclophosphamide and diets on sister chromatid exchange frequency of rats. Cytologia 51: 671-678.

Dubroff, L. S., Ward, O. C. and Jarowski, C. I. 1979. Effect of dietary lysine and tryptophan supplementation on growth rate and toxicity of barbiturates and ethanol in rats. J. Pharm. Sci. 68: 1554-1557.

Gebhart, E. 1969. Zur Beeinflussung der Wirkung von Myleran auf menschlichen Chromosomen durch LCystein. Mut. Res. 7: 254-257.

- 1970. Die Wirkung von L-Cystein auf die Aberrationsauslösung in menschlichen Chromosomen durch chemische Mutagene I. Dosis-Wirkungsbeziehungen bei Verwendung von Trenimon als Aberrationsinduktor. Humangenetik 10: 115-126.

- 1973a. Untersuchungen über die Beeinflussung der chromosomenschädigenden Aktivität von Trenimon an menschlichen Lymphocyten in vitro durch Aminosäuren. Humangenetik 18: 247-246.

- 1973b. Vergleichende Untersuchungen über die Beeinflussung der chromosomenschädigenden Aktivität von 8-Hydroxy-chinolinsulfat in menschlichen Lymphocyten in vitro durch Radioprotektoren und Aminosäuren. Mut. Res. 18: 353-361.

- and Becher, R. 1976. Comparative studies on the specificity of anticlastogenic action in human lymphocytes in culture. Human Genet. 32: 49-64.

- and Kappauf, H. 1980. The action of three anticlastogens on the induction of SCE by Trenimon and 8hydroxyquinolin sulfate in human lymphocyte cultures. Env. Mut. 2: 191-200.

Jarowski, C. I. and Pytelwski, R. 1975. Utility of fasting essential amino acid plasma levels in formulation of nutritionally adequate diets III. Lowering of rat serum cholesterol levels by lysine supplementation. J. Pharm. Sci. 64: 690.

Morrison, A. E., Middleton, E. J. and McLaughlin, J. M. 1961. Blood amino acid studies II. Effect on dietary lysine concentration, sex and growth rate in plasma free lysine and threonine levels in the rat, Canad. J. Biochem. Physiol. 39: 1657-1665.

Raja, P. K. and Jarowski, C. I. 1975. Utility of fasting essential amino acid palsma levels in formulation of nutritionally adequate diets IV. Lowering the human plasma cholesterol and triglyceride levels by lysine and tryptophan supplementation. J. Pharm. Sci. 64: 691-692.

Speit, G., Wick, C. and Wolf, M. 1980 . Induction of sister chromatid exchange by hydroxylamine, hydrazine and isoniazid and their inhibition by cysteine. Hum. Genet. 54: 155-158. 
-, Vogel, W. and Wolf, M. 1982. Characterization of sister chromatid exchange induction by hydrogen peroxide. Env. Mut. 4: 135-142.

Torre, G. M, Lynch, V. D. and Jarowski, C. I. 1981. Lowering blood urea nitrogen with amino acid supplementation. J. Pharm. Sci. 70: 114. 